INVESTIGACIÓN

\title{
Caracterización epidemiológica y social de las personas con discapacidad intelectual en Cuba
}

\author{
Epidemiological and social characterization of the mentally- \\ retarded people in Cuba
}

\author{
Marcia Cobas Ruíz, ${ }^{\text {I }}$ Eduardo Zacca Peña, ${ }^{\text {II }}$ Paulina Aracelis Lantigua Cruz, ${ }^{\text {II }}$ \\ Miriam Portuondo Sao, ${ }^{\text {IV }}$ Francisco Morales Calatayud, ${ }^{V}$ Emelia Icart \\ PereiravI $^{\text {VI }}$ \\ ILicenciada en Ciencias Sociales. Ministerio de Salud Pública. La Habana, Cuba. \\ ${ }^{\mathrm{II}}$ Doctor en Medicina. Doctor en Ciencias Médicas. Máster en Epidemiología. Profesor \\ Titular. Dirección Nacional de Estadística. Ministerio de Salud Pública. La Habana, \\ Cuba.

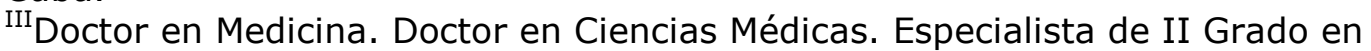 \\ Genética Clínica. Profesor Titular. Centro Nacional de Genética Médica. La Habana, \\ Cuba. \\ ${ }^{\mathrm{IV}}$ Doctor en Medicina. Máster en Genética Médica. Especialista de II Grado en \\ Genética Clínica. Centro Nacional de Genética Médica. La Habana, Cuba. \\ ${ }^{\vee}$ Doctor en Medicina. Doctor en Ciencias Médicas. Profesor Titular. Universidad de \\ Ciencias Médicas de La Habana. La Habana, Cuba. \\ ${ }^{\mathrm{VI}}$ Doctor en Medicina. Especialista en Pediatría. Centro Médico Psicopedagógico "La \\ Castellana". La Habana, Cuba.
}

\section{RESUMEN}

Objetivo Caracterizar desde el punto de vista epidemiológico y social a las personas con discapacidad intelectual en Cuba.

Método. El estudio interesó a toda la población cubana y dentro de ella a las personas con discapacidad intelectual como población diana. La pesquisa activa en la comunidad identificó, con el auxilio de los gobiernos locales y de las organizaciones sociales, a las personas registradas y a otras no registradas. Una visita al hogar de la persona con discapacidad permitió aplicar una combinación de entrevistas y exámenes clínicos que facilitaron el proceso de clasificación y registro de estos individuos.

Resultados Se estudiaron en todo el territorio nacional 140489 personas. La tasa de prevalencia de discapacidad intelectual en Cuba en el período estudiado era de

http://scielo.sld.cu 
1,25 por cada 100 habitantes. Las provincias con tasas más elevadas fueron Guantánamo y Santiago de Cuba. En el total de las personas estudiadas, la discapacidad intelectual fue más frecuente en el grupo de 15 a 19 años y hubo predominio de la discapacidad ligera. Se detectaron 29536 personas con incapacidad intelectual no registrados con anterioridad. La orfandad como factor social agravante en las personas con discapacidad intelectual tuvo muy baja frecuencia.

Conclusiones El procedimiento de pesquisa activa en la comunidad permitió el registro e inclusión en el estudio de aproximadamente una quinta parte de las personas con discapacidad intelectual no inscritas anteriormente. Los registros rutinarios de estas personas tienden a subestimar la real prevalencia de este problema de salud que es necesario conocer en toda su amplitud para poder atenderlo satisfactoriamente desde el punto de vista médico y social.

Palabras clave: Discapacidad intelectual, epidemiología, prevalencia, pesquisa activa, Cuba.

\begin{abstract}
Objective To characterize the mentally-retarded people in Cuba from the epidemiological and social viewpoints.

Methods The study covered the whole Cuban population, particularly those people with mental retardation taken as target population. The active screening carried out in the community allowed identifying the registered and non-registered persons with this condition, with the support of the local governments and social organizations. Home visits allowed using a combination of interviews and clinical tests to facilitate the process of classification and registration of these persons. Results A total of 140489 people were studied throughout the country. The prevalence rate of mental retardation in Cuba in the studied period was 1,25 per 100 pop. The provinces which exhibited the highest rates were Guantánamo and Santiago de Cuba. The mental retardation was more common in 15-19 years-old group, being slight retardation predominant. The study detected 29536 people with mental retardation, who had not been previously registered. Being an orphan as an aggravating social factor in mentally-retarded people was very uncommon.

Conclusions The active community-wide screening made it possible to identify and include in this study almost $20 \%$ of mentally-retarded persons no previouosly registered. The routine records of these people tend to underestimate the real prevalence of this health problem, which is required to be known in-depth in order to cope with it in a satisfactory way from the medical and social viewpoints.
\end{abstract}

Key words: Mental retardation, epidemiology, prevalence, active screening, Cuba.

\title{
INTRODUCCIÓN
}

La discapacidad intelectual aislada o asociada a malformaciones adicionales afecta entre el 2-3\% de la población general. A pesar del gran número de estudios que

http://scielo.sld.cu 
existen, muchas de las causas que originan el retraso mental aún se desconocen, y tan sólo se identifica su origen en el 50 \% de las personas afectadas.

Las causas genéticas y exógenas de la discapacidad intelectual se presentan con una frecuencia muy similar y con un rango muy variable, desde el 17,4 hasta el $47,1 \%{ }^{1}$

La discapacidad intelectual se manifiesta antes de los 18 años de edad y puede estar asociada, en algunos casos, a limitaciones físico-motoras y sensoriales, así como a trastornos conductuales.

Aunque la etiología de la discapacidad intelectual responde a factores genéticos o ambientales, definir cada uno de ellos es a veces muy difícil, ya que si bien los primeros son siempre de origen prenatal, los segundos pueden estar presentes en las etapas prenatal, perinatal y posnatal. ${ }^{2}$

Cuando los factores ambientales actúan en etapas muy tempranas del desarrollo embrionario (prenatal) las malformaciones congénitas resultan verdaderas fenocopias de defectos genéticos.

Si bien esta compleja madeja etiológica en la discapacidad intelectual resulta un inquietante reto, no menos complejo resulta el desafío de brindar una atención social inclusiva e integradora a estas personas en el marco de sus derechos como seres humanos.

Formando parte de una investigación de mayor extensión, se consideró pertinente incluir como una de las principales acciones, la caracterización epidemiológica y social de las personas con discapacidad intelectual.

\section{MÉTODOS}

Como parte de una investigación epidemiológica descriptiva de tipo transversal realizada en la República de Cuba en el período 2001-2003, la discapacidad intelectual fue una de las principales entidades consideradas. El estudio incluyó a toda la población cubana con discapacidad residente en el territorio nacional y dentro de ellas, las personas con discapacidad intelectual.

A los efectos de este artículo y de la medición, se consideró como discapacidad intelectual al nivel de funcionamiento intelectual significativamente inferior al de la media, que por lo general coexiste junto a limitaciones en dos o más áreas de habilidades adaptativas, tales como comunicación, autodirección, salud y seguridad, habilidades académicas funcionales, tiempo libre y trabajo.

En términos prácticos, la discapacidad se manifiesta en los siguientes niveles de funcionamiento:

\section{Ligera}

Posee potencialidades de escolaridad hasta noveno grado en educación especial.

- Susceptible de formación laboral.

http://scielo.sld.cu 
- Capaz de integrarse social y laboralmente.

\section{Moderada}

Posee potencialidades de escolaridad hasta sexto grado en educación especial.

- Susceptible de formación laboral.

- Capaz de integrarse social y laboralmente con ayuda.

\section{Severa}

Posee potencialidades para el logro de habilidades adaptativas funcionales.

- Susceptible de aprendizaje de técnicas laborales elementales.

- Capaz de integrarse social y laboralmente con ayuda en un medio protegido.

\section{Profunda}

Posee potencialidades de comunicación verbal o extraverbal elementales.

- Dependiente en actividades de la vida diaria.

- Sus posibilidades de desarrollo están directamente relacionadas con las oportunidades que les ofrecen tanto en la familia como en la comunidad. ${ }^{3}$

El universo inicial de personas con discapacidad fue obtenido de diversas fuentes, especialmente de registros censales y de asociaciones de personas con discapacidad, también de personas dispensarizadas por discapacidad en la atención primaria de salud, así como de otras instituciones de salud y educación.

Los listados con la localización de las personas con discapacidad fueron entonces ajustados en visitas efectuadas a las comunidades con el auxilio de las autoridades locales y de las organizaciones de masas.

Tres rondas de estudios pilotos, permitieron adecuar y validar dos instrumentos utilizados para registrar y clasificar a las personas con discapacidad intelectual.

La actividad de campo se caracterizó, en primer término, por la pesquisa activa de las personas con discapacidad intelectual en la comunidad y en segundo lugar, por la visita a sus hogares efectuada por un dúo integrado por un médico especialista en Medicina General Integral y un especialista en Psicopedagogía.

Siempre con el consentimiento informado de la persona con discapacidad o su tutor, les fue aplicada una combinación de entrevistas y exámenes clínicos como procedimiento de pesquisa y cuando fue indispensable, pruebas psicométricas y exámenes de laboratorio genético. También un equipo multidisciplinarios de

http://scielo.sld.cu 
profesionales y técnicos especializados, volvieron a visitar a la persona con discapacidad intelectual en su hogar y completaron su atención.

Los datos registrados en los instrumentos eran captados diariamente, usando para ello una aplicación informática desarrollada al efecto. Se desplegaron procesos de garantía de la calidad de los datos, tanto en el trabajo de campo por acciones sistemáticas de supervisión, como por rutinas de validación en la carga y procesamiento de los datos. Se construyeron distribuciones de frecuencias de variables de personas y lugar. Posteriormente se calcularon tasas de prevalencia usando como denominador la población media del período 2001-2003 para Cuba y sus territorios.

Al concluir el trabajo de campo, los datos se procesaron rápidamente y una información detallada de los resultados era comunicada a la comunidad y a sus líderes formales e informales.

La investigación permitió identificar además problemas y restricciones que afectaban las condiciones de vida de las personas con discapacidad intelectual, pero su solución y los diferentes procedimientos utilizados será motivo de próximas publicaciones.

\section{RESULTADOS}

Se estudiaron en Cuba 140489 personas con discapacidad intelectual, lo que representa una tasa de prevalencia de 1,25 por cada 100 habitantes (tabla 1 ).

Tabla 1. Tasa de prevalencia de discapacidad intelectual por provincias. Cuba 2001-2003

\begin{tabular}{|l|r|r|r|}
\hline Provincia & Población general & \multicolumn{1}{|c|}{ No. } & $\begin{array}{c}\text { Tasa } \\
\times 100 \text { hab. }\end{array}$ \\
\hline Pinar del Río & 739473 & 11260 & 1,52 \\
\hline La Habana & 717378 & 8355 & 1,16 \\
\hline Ciudad de La Habana & 2181395 & 13978 & 0,64 \\
\hline Matanzas & 665419 & 7283 & 1,09 \\
\hline Villa Clara & 836350 & 11124 & 1,33 \\
\hline Cienfuegos & 398569 & 5221 & 1,31 \\
\hline Sancti Spíritus & 463850 & 5427 & 1,17 \\
\hline Ciego de Ávila & 413447 & 6064 & 1,47 \\
\hline Camagüey & 791815 & 11398 & 1,44 \\
\hline Las Tunas & 532550 & 7123 & 1,34 \\
\hline Holguín & 1036097 & 16538 & 1,60 \\
\hline Granma & 836472 & 10134 & 1,21 \\
\hline Santiago de Cuba & 1041373 & 17171 & 1,65 \\
\hline Guantánamo & 516048 & 8492 & 1,65 \\
\hline Isla de la Juventud & 87850 & 921 & 1,05 \\
\hline Total & 11258086 & 140489 & 1,25 \\
\hline
\end{tabular}

Fuente: Estudio psicosocial y clínico genético de la discapacidad en Cuba. 
El estudio detectó 29536 personas con discapacidad intelectual en Cuba (21,02 $\%)$, no registrados con anterioridad.

Las provincias con tasas más elevadas fueron: Guantánamo y Santiago de Cuba, las que alcanzaron una tasa de prevalencia de 1,65 por 100 habitantes respectivamente, le siguieron en orden descendiente Holguín con una tasa 1,60 por 100 habitantes, Pinar del Río con 1,52 por 100 habitantes y Ciego de Ávila con 1,47 por 100 habitantes. El mayor número de personas con discapacidad intelectual se encontró en Santiago de Cuba (17 171), Holguín (16 538) y Ciudad de La Habana (13 978). Nueve provincias presentaron un comportamiento superior a la media nacional.

Las tasas de prevalencia específicas por grupo de edad de las personas con discapacidad intelectual (tabla 2), mostraron su mayor frecuencia en el grupo de 15 a 19 años, con una tasa de 2,30 por cada 100 habitantes, seguido del grupo de 20 a 29 años con valores de 1,70 por 100 habitantes y luego por el grupo de 5 a 14 años con tasa de prevalencia de 1,69 por cada 100 habitantes. El cuarto grupo de edades entre los de mayor frecuencia fue el grupo de 30 a 39 años con tasa de prevalencia de 1,40 por cada 100 habitantes. Estos cuatros grupos agruparon las tres cuartas parte del total de personas con discapacidad intelectual.

Tabla 2. Personas con discapacidad intelectual por grupos de edades. Cuba 2001-2003

\begin{tabular}{|l|r|r|r|}
\hline \multicolumn{1}{|c|}{ Grupos de edades } & No. & $\%$ & $\begin{array}{c}\text { Tasa } \\
\times 100 \text { hab. }\end{array}$ \\
\hline $0-4$ & 1751 & 1,2 & 0,24 \\
\hline $5-14$ & 27644 & 19,7 & 1,69 \\
\hline $15-19$ & 17834 & 12,7 & 2,30 \\
\hline $20-29$ & 29125 & 20,7 & 1,70 \\
\hline $30-39$ & 30479 & 21,7 & 1,40 \\
\hline $40-59$ & 25380 & 18,1 & 0,97 \\
\hline 60 y más & 8276 & 5,9 & 0,52 \\
\hline Total & 140489 & 100,0 & 1,25 \\
\hline
\end{tabular}

Fuente: Estudio psicosocial y clínico genético de la discapacidad en Cuba.

Las tasas de prevalencia de discapacidad intelectual en Cuba para cada sexo (tabla 3) se comportó de la siguiente manera: el masculino mostró una tasa de 1,49 por cada 100 habitantes del mismo sexo, mientras que el femenino solo alcanzó una prevalencia de 1,02 por cada 100 habitantes femeninas. La distribución porcentual de las personas con discapacidad intelectual en el varón fue de 59,4 contra 40,6 \% en las hembras.

La razón entre sexos (masculino/femenino) de 1,5 ilustra el predominio de la discapacidad intelectual en el sexo masculino.

http://scielo.sld.cu 
Tabla 3. Personas con discapacidad intelectual según sexo. Cuba 2001-03

\begin{tabular}{|l|c|c|c|}
\hline \multirow{2}{*}{ Sexo } & \multicolumn{3}{|c|}{ República de Cuba } \\
\cline { 2 - 3 } & No. & $\%$ & $\begin{array}{c}\text { Tasa } \\
\times 100 \text { hab. }\end{array}$ \\
\hline Femenino & 57034 & 40,6 & 1,02 \\
\hline Masculino & 83455 & 59,4 & 1,49 \\
\hline Razón M/F & 1,5 & & \\
\hline Total & 140489 & 100,0 & 1,25 \\
\hline
\end{tabular}

Fuente: Estudio psicosocial y clínico genético de la discapacidad en Cuba.

Cuando se consideró el grado de severidad de las personas con discapacidad intelectual (tabla 4), se observó un predominio de la forma ligera $(56,5 \%)$. La forma moderada fue la segunda categoría predominante.

Tabla 4. Personas con discapacidad intelectual según grado de intensidad de la discapacidad. Cuba. 2001-2003

\begin{tabular}{|l|r|r|r|}
\hline Grado de intensidad & No. & \multicolumn{1}{|c|}{$\%$} & $\begin{array}{c}\text { Tasa } \\
\times 100 \text { hab. }\end{array}$ \\
\hline Ligero & 79442 & 56,5 & 0,71 \\
\hline Moderado & 36558 & 26,0 & 0,32 \\
\hline Severo & 15548 & 11,1 & 0,13 \\
\hline Profundo & 8941 & 6,4 & 0,08 \\
\hline Total & 140489 & 100,0 & 1,21 \\
\hline
\end{tabular}

Fuente: Estudio psicosocial y clínico genético de la discapacidad en Cuba.

La investigación consideró otras características de interés en las personas con discapacidad intelectual (tabla 5). El $39 \%$ eran funcionalmente dependientes, el $29,5 \%$ no poseían control de esfínteres, el 3,3 \% eran encamados permanentes;

$20,8 \%$ vivían en una vivienda en mal estado y el $15,4 \%$ lo hacían en una vivienda en estado crítico. El 37,5\% tenía antecedente de un familiar cercano con discapacidad intelectual, la frecuencia de consanguinidad se presentó en el 5,2 \%. Solo el 3,5\% de las personas con discapacidad intelectual tenían síndrome de Down. La orfandad como factor social agravante en las personas con discapacidad intelectual tuvo una muy baja frecuencia. 
Tabla 5. Personas con discapacidad intelectual según algunas caracteristicas. Cuba 2001-2003. $n=140489$

\begin{tabular}{|l|r|r|}
\hline Características & No. & \multicolumn{1}{|c|}{$\%$} \\
\hline Dependencia funcional & 54746 & 39,0 \\
\hline Pérdida del control de esfinteres & 41452 & 29,5 \\
\hline Encamamiento & 4698 & 3,3 \\
\hline Vivienda en mal estado & 29277 & 20,8 \\
\hline Vivienda en estado critico & 21692 & 15,4 \\
\hline Antecedente de familiar cercano con discapacidad intelectual & 52720 & 37,5 \\
\hline Antecedente de consanguinidad & 7323 & 5,2 \\
\hline Síndrome de Down & 4919 & 3,5 \\
\hline Orfandad de madre & 1139 & 0,81 \\
\hline Orfandad de padre & 1866 & 1,3 \\
\hline Orfandad ambos padres & 416 & 0,3 \\
\hline
\end{tabular}

Fuente: Estudio psicosocial y clínico genético de la discapacidad en Cuba.

\section{DISCUSIÓN}

Resulta interesante señalar que los estudiosos del tema a lo largo de los últimos 50 años, señalan que la prevalencia de la discapacidad intelectual no ha sufrido grandes cambios, debido al balance entre los avances médicos, educacionales y la emergencia de nuevas enfermedades, o factores que traen consigo aparejados el aumento de esta discapacidad. Por ejemplo, el decrecimiento del síndrome de Down puede relacionarse con la implementación del diagnóstico prenatal citogenético, o la disminución de la discapacidad intelectual severa por enfermedades como la fenilcetonuria tratada precozmente u otros defectos metabólicos diagnosticados por pesquisajes masivos neonatales y consecuentemente la aplicación de tratamientos tempranos, sin embargo, la prevalencia ha sido contrabalanceada por el aumento de síndromes como el fetal alcohólico, el abuso de drogas en el período de gestación o el síndrome de inmunodeficiencia adquirido (sida) congénito.

Del mismo modo, ha decrecido marcadamente la mortalidad de niños prematuros con peso entre 1500 y $2500 \mathrm{~g}$, y esto ha aumentando la supervivencia de niños muy prematuros, quienes luego exhiben un mayor rango de morbilidad para los trastornos severos del sistema nervioso central. ${ }^{4}$ De esta manera se mantienen las cifras de prevalencia entre el 1 y el $3 \%$, de forma general. ${ }^{5}$

Como se describe en estudios internacionales, el exceso de varones afectados por la discapacidad intelectual se observa en todos los estudios en diferentes países. ${ }^{2}$ Esto tiene una explicación genética, ya que se han identificado más de 200 genes localizados en el cromosoma $X$ que se relacionan a su vez con la inteligencia y es en el varón donde siempre se expresa este cromosoma.

La elección de una pareja o cónyuge y el proceso de endogamia o exogamia son fenómenos multidimensionales, donde se combinan factores culturales, demográficos y psicosociales, ${ }^{6}$ por lo que la frecuencia de consanguinidad tiene valores diferentes de acuerdo a las características de la población de cada zona.

http://scielo.sld.cu 
De igual manera en el estudio de la discapacidad intelectual se analizó la presencia de familiares de primer grado con esta discapacidad por tratarse la inteligencia de un rasgo cuantitativo que se expresa a partir de la acción aditiva de varios genes cuya expresión puede modificarse por la acción de factores ambientales, en beneficio o detrimento. Este aspecto sugiere mayor predisposición genética en estas familias para desarrollar discapacidad intelectual ante condiciones ambientales adversas.

La discapacidad intelectual, puede ser un componente de un síndrome más complejo (síndrome Frágil X, Down y otros), síntoma en un desorden metabólico, o un fenotipo exclusivamente, que afecta solo el desarrollo posnatal de la función cognitiva, y puede ser causada por factores genéticos, ambientales o ecogenéticos, como expresión del disturbio de la proliferación neuronal, migración, diferenciación, crecimiento axonal, formación de las sinapsis, arborización dendrítica o su proceso de eliminación, como resultado de la disrupción genética y su expresión molecular, o del insulto ambiental, e incluso en algunos casos, de la conjugación de ambos. ${ }^{7}$ Como regla, en más del $50 \%$ de los casos la causa permanece sin determinarse. ${ }^{8-}$ 10

Se puede concluir que aproximadamente una quinta parte de las personas con discapacidad intelectual estudiadas no habían sido registradas con anterioridad, fue a través de la pesquisa activa en la comunidad, el procedimiento que permitió su inclusión en el estudio. Los registros rutinarios de estas personas tienden a subestimar la real prevalencia de este problema de salud que es necesario conocer en toda su amplitud para poder atenderlo satisfactoriamente desde el punto de vista médico y social.

\section{REFERENCIAS BIBLIOGRÁFICAS}

1. Flint J, Knight S. The use of telomere probes to investigate submicroscopic rearrangements associated with mental retardation. Curr Opin Genet Dev. $2003 ; 13: 10-6$.

2. Clasificación de los factores causales de retraso mental. En: Colectivo de autores, editores. Por la Vida. Estudio clínico genético de las personas con retraso mental. La Habana: Casa Editora Abril; 2003. p. 115-6.

3. Definiciones de discapacidad y criterios de inclusión y exclusión en el estudio. En: Colectivo de autores, editores. Por la Vida. Estudio clínico genético de las personas con retraso mental. La Habana: Casa Editora Abril; 2003. p. 87-9.

4. Batshaw Mark L. Mental Retardation. Pediatric Clinics of North America. 1993;40(3):507-21.

5. Portuondo Sao M. Estudio epidemiológico del retraso mental en el policlínico "Carlos J. Finlay" de 1977 a 1997 [tesis]. La Habana: Centro Nacional de Genética Médica; 2001.

6. Rodríguez García D. Endogamia, exogamia y relaciones interétnicas [tesis]. Barcelona: Universidad Autónoma de Barcelona; 2002.

7. Harvey B. Sarnat, Menkes JH. Child Neurology. Lippincott: Williams \& Wilkins; 2000.

http://scielo.sld.cu 
8. Lewis RA. Retardo mental. Información general. University of Maryland Medical Center [sitio en Internet]. [citada 30 Oct 2006]. Disponible en: http://www.umm.edu/esp_ency/article/001523.htm

9. Rodríguez Revenga Bodi L. Retraso mental de origen genético. Rev Neurol [serie en Internet]. 2006 [citada 30 Oct 2006];43. Disponible en:

http://www.neurologia.com/sec/resumen.php?id=2006419

10. Guitart-Feliubadaló M. Causas cromosómicas que originan el retraso mental: alteraciones cromosómicas diagnosticables en el paciente. Rev Neurol [serie en Internet]. 2006[citada 30 Mar 2010];42(Supl. 1). Disponible en:

http://www.sld.cu/galerias/pdf/sitios/rehabilitacion-

logo/retraso mental cromosomico.pdf

Recibido: 27 de junio de 2010.

Aprobado: 31 de julio de 2010.

Marcia Cobas Ruíz. Viceministra del Ministerio de Salud Pública. Ministerio de Salud Pública. Calle 23 No. 201 e/ M y N. El Vedado 10400, Plaza de la Revolución. La Habana, Cuba. Correo electrónico: marciacobas@infomed.sld.cu;

zacca@infomed.sld.cu 